\title{
Radionic nonuniform black strings
}

$\operatorname{AUTHOR}(\mathrm{S})$ :

Tamaki, T; Kanno, S; Soda, J

\section{CITATION:}

Tamaki, T ...[et al]. Radionic nonuniform black strings. PHYSICAL REVIEW D 2004, 69(2): 024010.

ISSUE DATE:

2004-01

URL:

http://hdl.handle.net/2433/49869

RIGHT:

Copyright 2004 American Physical Society 
PHYSICAL REVIEW D 69, 024010 (2004)

\title{
Radionic nonuniform black strings
}

\author{
Takashi Tamaki, ${ }^{*}$ Sugumi Kanno ${ }^{\dagger}$ and Jiro Soda \\ Department of Physics, Kyoto University, 606-8501, Japan
}

(Received 5 August 2003; published 29 January 2004)

\begin{abstract}
Nonuniform black strings in the two-brane system are investigated using the effective action approach. It is shown that the radion acts as a nontrivial hair of the black strings. From the brane point of view, the black string appears as the deformed dilatonic black hole which becomes a dilatonic black hole in the single brane limit and reduces to the Reissner-Nordstrom black hole in the close limit of two-branes. The stability of solutions is demonstrated using catastrophe theory. From the bulk point of view, the black strings are proved to be nonuniform. Nevertheless, the zeroth law of black hole thermodynamics still holds.
\end{abstract}

DOI: 10.1103/PhysRevD.69.024010

PACS number(s): 04.40.-b, 04.50.+h, 04.70.-s

\section{INTRODUCTION}

Recent progress in superstring theory has suggested the possibility that we reside on a hypersurface in higherdimensional space-time [1]. This so-called brane world scenario has rapidly become popular since Randall and Sundrum proposed simple models in which we live on a brane embedded in $\mathrm{AdS}_{5}$ space-time [2]. There are two types of Randall-Sundrum (RS) scenarios. In the first RS model (RS1), the two flat branes are embedded in $\mathrm{AdS}_{5}$ space-time to solve the hierarchy problem. In the second RS model (RS2), one positive tension brane is embedded in $\operatorname{AdS}_{5}$ space-time.

The cosmology and black holes in RS models have been investigated actively [3-10]. The common issue is to understand the effects of the bulk geometry on the brane world. The radion and Kaluza-Klein modes are key ingredients for this aim. Here, it should be noted that most of the important phenomena occur at low energies or the low-curvature regime. At low energies, the radion would dominate the effects of the extra dimension. In the case of the RS2 model, however, the radion does not exist. Hence, except for a small contribution from the Kaluza-Klein modes, the conventional Einstein theory is recovered at low energies in the RS2 model. On the other hand, in the case of the RS1 model, the existence of the radion is crucial. Recently, we have developed a systematic method to study a brane world at low energies using the gradient expansion method [11]. In particular, we have shown that gravity in the RS1 model can be described by a four-dimensional scalar-tensor theory and the information of the bulk can be reconstructed through holograms. The cosmology has been investigated using this approach and found to be an interesting scenario [12]. As our method is quite general, it is also applicable to black hole physics.

In conventional general relativity, the Schwarzschild solution is the simplest stable black hole solution, while in superstring theory dilatonic black holes have been found by Gibbons and Maeda and independently by Garfinkle, Horo-

\footnotetext{
*Electronic address: tamaki@tap.scphys.kyoto-u.ac.jp

${ }^{\dagger}$ Electronic address: sugumi@tap.scphys.kyoto-u.ac.jp

ॠElectronic address: jiro@tap.scphys.kyoto-u.ac.jp
}

witz, and Strominger (GM-GHS) [13]. Interestingly, dilatonic black holes are stable in the same sense as a Schwarzschild black hole [14]. In this sense, these are realistic black holes in superstring theory. Let us go back to the brane world. The conventional Schwarzschild black hole can be realized as a section of the uniform black string. However, this is not a unique possibility. The black hole can be localized on the brane, although it may not appear as an exact Schwarzschild black hole. Still, to find all of the stable black holes in the brane world is an open issue. From the string theoretic point of view, it is also desirable to find GM-GHSlike solutions in the brane world scenario. Unfortunately, no exact black string solution is known in this case. Therefore, it is interesting to seek black strings corresponding to GMGHS black holes in RS models.

In the RS2 model, it is argued that the black hole should be localized on the brane because of "Gregory-Laflamme" instability of the black string [15]. These localized black holes have been investigated intensively [4-9]. On the other hand, in the RS1 model, the black string exists without suffering from the Gregory-Laflamme instability if the distance between two branes is less than the radius of the black hole on the brane. In the opposite condition, Gregory-Laflamme instability commences. Intriguingly, since the dynamics of the radion controls the length of the black string, it can trigger the transition from the stable to unstable black string. It is argued that the fate of the unstable black string is either the localized black hole or the nonuniform black string [16-18]. Clearly, the first step to this direction is to understand the role of the radion in the black strings. Although numerical analysis is practical to attack this issue, the effort must be made toward the analytic understanding of the phenomena.

The purpose of this paper is to present a semianalytic approach to investigate black strings in the RS1 model. We investigate the black strings corresponding to GM-GHS black holes on the brane using the proposed method. In particular, the role of the radion in the nonuniform black strings is explored.

This paper is organized as follows. In Sec. II, we briefly review our effective action approach. In Sec. III, we describe basic equations for the numerical analysis. In Sec. IV, we describe our solution from the four-dimensional point of view with an emphasis on the role of the radion. In Sec. V, 
we view our solution from the bulk. In Sec. VI, we summarize our results.

\section{EFFECTIVE ACTION APPROACH}

We begin by reviewing the low-energy effective action derived in previous papers $[11,19]$. We consider an $S_{1} / Z_{2}$ orbifold space-time with the two branes as the fixed points. In the RS1 model, the positive $(\oplus)$ and negative $(\ominus)$ tension branes are embedded in $\mathrm{AdS}_{5}$ with curvature radius $\ell$. Our system is described by the action

$$
\begin{aligned}
S= & \frac{1}{2 \tilde{\kappa}^{2}} \int d^{5} x \sqrt{-g}\left(\mathcal{R}+\frac{12}{\ell^{2}}\right)-\sum_{i=\oplus, \ominus} \sigma_{i} \int d^{4} x \sqrt{-g^{i \text {-brane }}} \\
& +\sum_{i=\oplus, \ominus} \int d^{4} x \sqrt{-g^{i \text {-brane }}} \mathcal{L}_{\text {matter }}^{i}
\end{aligned}
$$

where $\mathcal{R}, g_{\mu \nu}^{i \text {-brane }}, \sigma_{i}$, and $\widetilde{\kappa}^{2}$ are the five-dimensional scalar curvature, the induced metric on the $i$-brane, the tension on the $i$-brane, and the five-dimensional gravitational constant, respectively. Here, we assume the relations $\sigma_{\oplus}=6 /\left(\tilde{\kappa}^{2} \ell\right)$ and $\sigma_{\ominus}=-6 /\left(\tilde{\kappa}^{2} \ell\right)$.

For general nonflat branes, we cannot keep both the two branes straight in the Gaussian normal coordinate system. Hence, we use the following coordinate system to describe the geometry of the brane model:

$$
d s^{2}=e^{2 \eta\left(x^{\mu}\right)} d y^{2}+g_{\mu \nu}\left(y, x^{\mu}\right) d x^{\mu} d x^{\nu} .
$$

We place the branes at $y=0(\oplus$-brane $)$ and $y=\ell(\ominus$-brane $)$ in this coordinate system. In this coordinate system, the proper distance between two branes with fixed $x^{\mu}$ is represented as

$$
d\left(x^{\mu}\right)=\int_{0}^{\ell} e^{\eta\left(x^{\mu}\right)} d y
$$

Hence, we call $\eta$ the radion.

The strategy we take in this paper is the following. Let us start with the variation of the action

$$
\delta S\left[g_{\mu \nu}, h_{\mu \nu}^{i}\right]=\frac{\delta S}{\delta g_{\mu \nu}} \delta g_{\mu \nu}+\sum_{i=\oplus, \ominus} \frac{\delta S}{\delta h_{\mu \nu}^{i}} \delta h_{\mu \nu}^{i}=0 .
$$

The variation with respect to $g_{\mu \nu}$ gives the bulk Einstein equations and the variation with respect to $h_{\mu \nu}$ yields the junction conditions. First, we solve the bulk equations of motion with $h_{\mu \nu}^{i}$ fixed; then, we get the relation

$$
g_{\mu \nu}=g_{\mu \nu}\left[h_{\mu \nu}^{i}\right] .
$$

Substituting Eq. (5) into junction conditions, we get the fourdimensional effective equations of motion for the induced metric $h_{\mu \nu}^{i}$. Or, by substituting Eq. (5) into the action, we obtain four-dimensional effective action

$$
S_{\mathrm{eff}}=S\left[g_{\mu \nu}\left[h_{\mu \nu}^{i}\right], h_{\mu \nu}^{i}\right]
$$

From Eq. (6), the four-dimensional effective equations of motion can be reproduced.

Of course, we must resort to some approximation method to solve bulk equations of motion. As the usual phenomena occur at low energies

$$
\frac{\rho_{i}}{\left|\sigma_{i}\right|} \sim \frac{\tilde{\kappa}^{2} \rho_{i}}{\tilde{\kappa}^{2}\left|\sigma_{i}\right|} \sim\left(\frac{\ell}{L}\right)^{2} \ll 1,
$$

where $L$ denotes the characteristic length scale of the curvature on the brane, we can employ the gradient expansion method [20]. We seek the metric as a perturbative series with the number of derivatives increasing with the order of iteration-that is, $O\left((\ell / L)^{2 n}\right), n=0,1,2, \ldots$-as

$$
\begin{aligned}
g_{\mu \nu}\left(y, x^{\mu}\right) & =b^{2}(y, x)\left[h_{\mu \nu}\left(x^{\mu}\right)+g_{\mu \nu}^{(1)}\left(y, x^{\mu}\right)+\cdots\right] \\
b(y, x) & =\exp \left(-y e^{\eta} / \ell\right)
\end{aligned}
$$

where we put the Dirichlet boundary condition $g_{\mu \nu}(y$ $=0, x)=h_{\mu \nu}(x)$ at the $\oplus$-brane. Substituting Eq. (8) into the bulk Einstein equations, we can obtain the relation (5) perturbatively. The first order correction is given by

$$
\begin{aligned}
g_{\mu \nu}^{(1)}= & -\frac{\ell^{2}}{2}\left[\left(\frac{1}{b^{2}}-1\right)\left(R_{\mu \nu}-\frac{1}{6} h_{\mu \nu} R\right)\right. \\
& -\left(\frac{1}{b^{2}}-1-\frac{2 y e^{\eta}}{\ell} \frac{1}{b^{2}}\right)\left(\eta_{\mid \mu \nu}+\frac{h_{\mu \nu}}{2} \eta^{\mid \alpha} \eta_{\mid \alpha}\right) \\
& \left.+\frac{2 y^{2} e^{2 \eta}}{\ell^{2} b^{2}}\left(\eta_{\mid \mu} \eta_{\mid \nu}-\frac{h_{\mu \nu}}{2} \eta^{\mid \alpha} \eta_{\mid \alpha}\right)+\left(\frac{1}{b^{4}}-1\right) \chi_{\mu \nu}\right]
\end{aligned}
$$

where

$$
\begin{aligned}
\chi_{\nu}^{\mu}= & -\frac{\kappa^{2}(1-\Psi)}{2 \Psi}\left\{T_{\nu}^{\oplus \mu}+(1-\Psi) T^{\ominus \mu}{ }_{\nu}\right\} \\
& -\frac{1}{2 \Psi}\left[\left(\Psi^{\mid \mu}{ }_{\mid \nu}-\delta_{\nu}^{\mu} \Psi_{\mid \alpha}^{\mid \alpha}\right)\right. \\
& \left.+\frac{3}{2(1-\Psi)}\left(\Psi^{\mid \mu} \Psi_{\mid \nu}-\frac{1}{2} \delta_{\nu}^{\mu} \Psi^{\mid \alpha} \Psi_{\mid \alpha}\right)\right]
\end{aligned}
$$

is nothing but the projected Weyl tensor $E_{\mu \nu}[21]$. Here, $\kappa^{2}$ $:=\tilde{\kappa}^{2} / \ell$ and

$$
\Psi:=1-\exp \left(-2 e^{\eta}\right)
$$

is defined for convenience. We also call $\Psi$ the radion. The junction condition gives the effective equations of motion from which the action for the $\oplus$-brane can be read off as

$$
\begin{aligned}
S_{\oplus}= & \frac{1}{2 \kappa^{2}} \int d^{4} x \sqrt{-h}\left[\Psi R(h)-\frac{3}{2(1-\Psi)} \Psi^{\mid \alpha} \Psi_{\mid \alpha}\right] \\
& +\int d^{4} x \sqrt{-h} \mathcal{L}^{\oplus}+\int d^{4} x \sqrt{-h}(1-\Psi)^{2} \mathcal{L}^{\ominus} .
\end{aligned}
$$


If we can obtain a solution for this system (13), the bulk metric corresponding to the 4-dimensional effective theory is given by

$$
g_{\mu \nu}=(1-\Psi)^{y / \ell}\left[h_{\mu \nu}(x)+g_{\mu \nu}^{(1)}\left(h_{\mu \nu}, \Psi, T_{\mu \nu}^{\oplus}, T_{\mu \nu}^{\ominus}, y\right)\right],
$$

which corresponds to Eq. (5). Thus the bulk metric is completely determined by the energy-momentum tensors on both branes, the radion and the induced metric on the $\oplus$-brane. Therefore, once the four-dimensional solution of the quasiscalar-tensor gravity is given, one can reconstruct the bulk geometry from these data. The quasi-scalar-tensor gravity (13) works as holograms at the low energy.

Using the low-energy expansion scheme, in principle, we can calculate the higher-order corrections and perform the holographic reconstruction of the bulk metric. In the lowenergy regime which is our main concern, however, these corrections do not change our qualitative conclusion.

\section{BASIC EQUATIONS FOR NUMERICAL ANALYSIS}

Below, we consider a dilaton field coupled to the electromagnetic field on the $\oplus$-brane since it appears in the compactification process. Then, we have

$$
\begin{aligned}
S_{\oplus}= & \frac{1}{2 \kappa^{2}} \int d^{4} x \sqrt{-h}\left[\Psi R(h)-\frac{3}{2(1-\Psi)} \Psi^{\mid \alpha} \Psi_{\mid \alpha}\right] \\
& -\int d^{4} x \sqrt{-h}\left[\frac{1}{2} \phi^{\mid \alpha} \phi_{\mid \alpha}+\frac{1}{4} e^{-2 a \phi} F_{\mu \nu} F^{\mu \nu}\right],
\end{aligned}
$$

where $\phi$ and $a$ are a dilaton field and its coupling constant, respectively. Since our theory is a scalar-tensor-type theory, we call this original action the Jordan-frame effective action. In order to discuss the property of the radion, it is sometimes convenient to move to the Einstein frame in which the action takes the canonical Einstein-scalar form. Applying a conformal transformation $h_{\mu \nu}=(1 / \Psi) q_{\mu \nu}$ and introducing a new field

$$
\psi=-\sqrt{\frac{3}{2 \kappa^{2}}} \log \left|\frac{\sqrt{1-\Psi}-1}{\sqrt{1-\Psi}+1}\right|,
$$

we obtain the Einstein-frame effective action

$$
\begin{aligned}
S_{\oplus}= & \int d^{4} x \sqrt{-q}\left[\frac{1}{2 \kappa^{2}} R(q)-\frac{1}{2}(\nabla \psi)^{2}\right. \\
& \left.-\frac{1}{2}\left(\cosh ^{2} \frac{\kappa}{\sqrt{6}} \psi\right)(\nabla \phi)^{2}-\frac{1}{4} e^{-2 a \phi} F^{2}\right],
\end{aligned}
$$

where $\nabla$ denotes the covariant derivative with respect to the metric $q_{\mu \nu}$. We also call $\psi$ the radion. We take this action and consider the static and spherically symmetric metric as

$$
d s^{2}=-f(r) e^{-2 \delta(r)} d t^{2}+f(r)^{-1} d r^{2}+r^{2} d \Omega^{2},
$$

where $f(r):=1-\kappa^{2} m(r) / 4 \pi r$. We construct a black hole on the brane and construct a black string using holograms (14). We choose a vector potential $A_{\mu}$ as

$$
A_{\mu}=\left(0,0,0, Q_{m} \cos \theta\right),
$$

where $Q_{m}$ is a magnetic charge. If we also consider an electric charge, our system has an electric-magnetic duality $F$ $\rightarrow e^{-2 a \phi} \widetilde{F}, \phi \rightarrow-\phi$, where $\widetilde{F}$ is the Hodge dual of $F$. Hence we can easily obtain the results for the electrically charged case. Our basic equations are

$$
\begin{aligned}
m^{\prime}= & 4 \pi\left(\frac{r^{2}}{2} f U+\frac{Q_{m}^{2} e^{-2 a \phi}}{2 r^{2}}\right), \\
\delta^{\prime}= & -\frac{\kappa^{2}}{2} r U, \\
\phi^{\prime \prime}= & -\frac{2 \kappa}{\sqrt{6}} \psi^{\prime} \phi^{\prime} \tanh \left(\frac{\kappa}{\sqrt{6}} \psi\right) \\
& -\frac{1}{f}\left[\phi^{\prime} V+\frac{a e^{-2 a \phi} Q_{m}^{2}}{r^{4} \cosh ^{2}\left(\frac{\kappa}{\sqrt{6}} \psi\right)}\right], \\
\psi^{\prime \prime}= & -\frac{\psi^{\prime} V}{f}+\frac{\kappa}{\sqrt{6}}\left(\phi^{\prime}\right)^{2} \cosh \left(\frac{\kappa}{\sqrt{6}} \psi\right) \sinh \left(\frac{\kappa}{\sqrt{6}} \psi\right),
\end{aligned}
$$

where the prime denotes the derivative with respect to $r$ and

$$
\begin{aligned}
& U:=\left(\psi^{\prime}\right)^{2}+\left(\cosh ^{2} \frac{\kappa}{\sqrt{6}} \psi\right)\left(\phi^{\prime}\right)^{2}, \\
& V:=\frac{2}{r}-\frac{\kappa^{2} m}{4 \pi r^{2}}-\frac{\kappa^{2} Q_{m}^{2}}{2 r^{3}} e^{-2 a \phi} .
\end{aligned}
$$

We assume the existence of a regular event horizon at $r$ $=r_{H}$. So we have

$$
\begin{gathered}
m_{H}=\frac{4 \pi r_{H}}{\kappa^{2}}, \delta_{H}, \phi_{H}, \psi_{H}<\infty, \psi_{H}^{\prime}=0, \\
\phi_{H}^{\prime}=-\frac{a e^{-2 a \phi_{H}} Q_{m}^{2}}{V_{H} r_{H}^{4} \cosh ^{2}\left(\frac{\kappa}{\sqrt{6}} \psi_{H}\right)} .
\end{gathered}
$$

The variables with subscript $H$ are evaluated at the horizon. The boundary conditions at spatial infinity to guarantee asymptotic flatness are 


$$
\begin{aligned}
& m(\infty)=: M=\text { const }, \quad \delta(\infty)=0, \\
& \phi(\infty)=0, \quad \psi(\infty)=: \psi_{\infty}=\text { const }
\end{aligned}
$$

where $M$ corresponds to a gravitational mass of the black hole. We will obtain the black hole solutions numerically by solving Eqs. (20)-(23) iteratively with boundary conditions (26), (27), and (28).

\section{VIEW FROM THE BRANE}

The radion is affected by the matter on the brane and varies depending on the place on the brane. The other way around, the configuration of matter is affected by the radion. Hence, the radion must play an important role in the system of black string. As to the GM-GHS black holes, their stability is already known. It would be interesting to investigate if the radion destroys the stability or not.

\section{A. Radion as a hair}

If we include the moduli fields, the first law of black hole thermodynamics has the term depending on the scalar charge [22]. A contribution from both the dilaton field and radion field can be expected. We shall examine it starting from the action (17). Here, we consider the spherically symmetric and magnetically charged cases for simplicity. We denote the time translational Killing vector as $k:=\partial / \partial t$ and the null vector orthogonal to the event horizon $H$ as $n_{\mu}$ which is normalized as

$$
n_{\mu} k^{\mu}=-1
$$

We represent the volume element and the surface element as $d \sigma_{\mu}$ and $d S_{\mu \nu}$, respectively. Then, the gravitational mass $M$ and the "magnetic charge" $Q_{m}$ can be defined as

$$
\begin{gathered}
M:=\frac{-1}{4 \pi} \int_{\infty} k^{\mu ; \nu} d S_{\mu \nu}, \\
Q_{m}:=-\int_{\infty} e^{-2 a \phi} F^{\mu \nu} d S_{\mu \nu} .
\end{gathered}
$$

We can obtain the mass formula using the standard procedure [23] as

$$
\begin{aligned}
M= & \frac{1}{\kappa^{2}} \int\left[R-(\nabla \psi)^{2}-\cosh ^{2}\left(\frac{\kappa}{\sqrt{6}} \psi\right)(\nabla \phi)^{2}\right. \\
& \left.-\frac{1}{2} e^{-2 a \phi} F^{2}\right] k^{\mu} d \sigma_{\mu}+\frac{T_{H}}{2} A+2 \Phi_{H} Q_{H},
\end{aligned}
$$

where $T_{H}, \Phi_{H}$, and $Q_{H}$ are the Hawking temperature, the magnetic potential at the horizon, and the "magnetic charge" at the horizon, respectively. Taking the variation of this equation, we obtain the first law of black hole thermodynamics:

$$
\delta M=T_{H} \frac{\delta A}{4}+\Phi_{H} \delta Q_{H}-\Sigma \delta \psi,
$$

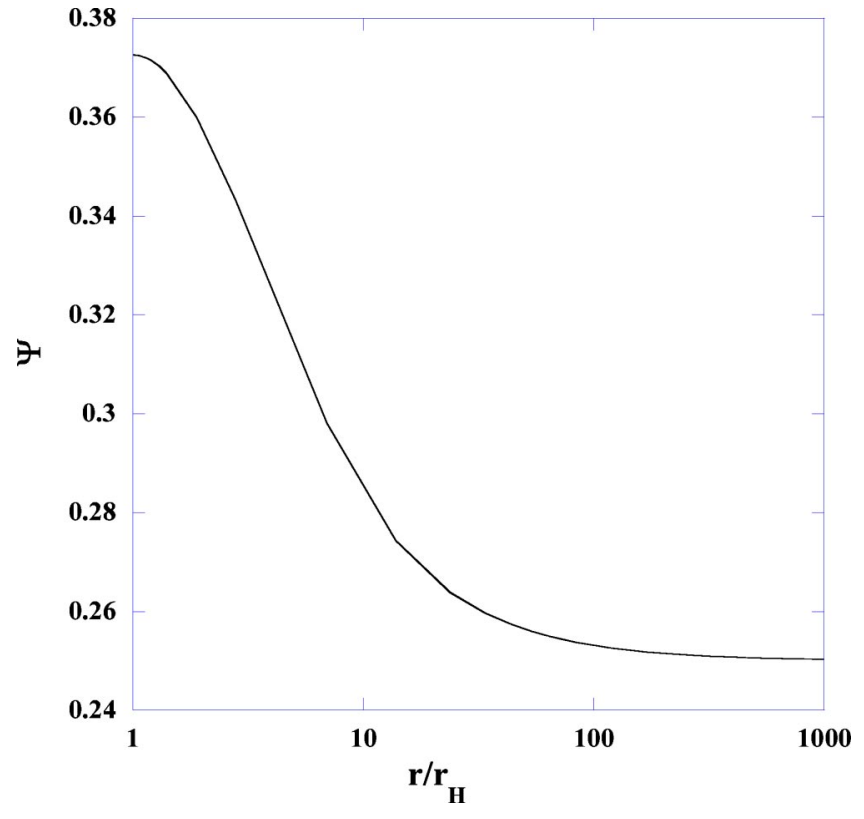

FIG. 1. The behavior of $\Psi$ for $\Psi_{\infty}=0.25, \lambda_{H}=0.119$, and $\bar{a}$ $=\sqrt{3}$.

where we defined the radionic charge $\Sigma$ as

$$
\Sigma:=2 \int_{\infty} \psi^{; \mu} k^{\alpha} d S_{\alpha \mu} .
$$

This formula shows that the dilatonic charge cannot be a true hair, because it is absorbed into the definition of the "magnetic charge" [24]. However, the radion field is a hair of black strings. To derive Eq. (33), we used the property that $\psi$ asymptotically behaves as $O(1 / r)$ which can be confirmed by asymptotic analysis of Eqs. (20)-(23). We can prove that $\psi(\Psi)$ is a monotonically increasing (decreasing) function of $r$ from Eq. (23). In Fig. 1, as an example, $\Psi$ for a solution $\bar{a}:=\sqrt{2} a / \kappa=\sqrt{3}, \Psi_{\infty}:=\Psi(\infty)=0.25$, and the horizon radius $\lambda_{H}:=\sqrt{2} r_{H} / \kappa Q_{m}=0.119$ is depicted [25].

\section{B. Stability of the black string}

First, we summarize the relation between $\Psi$ (or $\psi$ ) and $d$ using Eqs. (12) and (16) in Table I. $\Psi(\psi)$ is a monotonically increasing (decreasing) function with respect to $d$.

In the single brane limit $\psi \rightarrow 0$, the effect of the radion ceases. Hence, the solution approaches the GM-GHS solution. In the close limit $\psi \rightarrow \infty$, we have $\phi(r)=0$ from Eqs. (22) and (27). Thus, only Reissner-Nordström (RN) solutions are possible in this limit because of the no-hair theorem [26]. Therefore, we expect that the nontrivial radion $(\psi \neq 0, \infty)$ in-

TABLE I. Relation between variables.

\begin{tabular}{cccc}
\hline \hline & Close limit & & Single brane limit \\
\hline$d$ & 0 & $\rightarrow$ & $\infty$ \\
$\psi$ & $\infty$ & $\rightarrow$ & 0 \\
$\Psi$ & 0 & $\rightarrow$ & 1 \\
\hline \hline
\end{tabular}




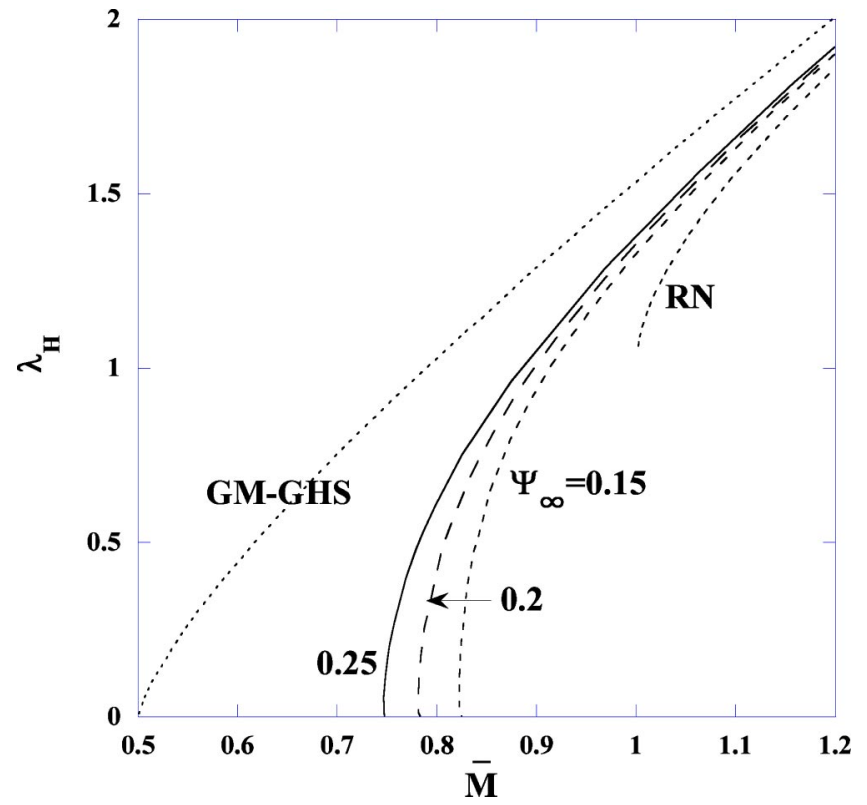

FIG. 2. The relation between the horizon radius $\lambda_{H}$ and the gravitational mass $\bar{M}$ for $\bar{a}=\sqrt{3}$.

terpolates RN and GM-GHS solutions.

As an illustration, we show the relation between the horizon radius $\lambda_{H}$ and the gravitational mass $\bar{M}$ $:=\kappa M / 4 \pi \sqrt{2} Q_{m}$ in Fig. 2. In fact, in the single brane limit, the solution approaches GM-GHS and the effect of the dilaton field fades away in the close limit.

We also notice that solutions exist until $\lambda_{H} \rightarrow 0$ except for the RN solution. This suggests that there is no inner horizon. Indeed, we can prove it. First, rewrite Eq. (22) as

$$
\left[f r^{2} e^{-\delta} \cosh ^{2}\left(\frac{\kappa}{\sqrt{6}} \psi\right) \phi^{\prime}\right]^{\prime}=\frac{a}{r^{2}} e^{-2 a \phi} e^{-\delta} Q_{m}^{2} .
$$

It follows that $D:=f r^{2} e^{-\delta} \cosh ^{2}[(\kappa / \sqrt{6}) \psi] \phi^{\prime}$ monotonically increases with $r$ for $a>0$. Hence, $D<0$ holds inside the horizon and then $f$ cannot become 0 , which implies that there is no inner horizon. Thus, the causal structure is the same as that of Schwarzschild black hole. This suggests that our solutions are as stable as the GM-GHS solutions [14].

We can also argue stability of our solutions using catastrophe theory. In Fig. 3, the relation between the inverse temperature $1 / \bar{T}_{H}:=\left(1 / T_{H}\right) /\left(\kappa Q_{m} / 2\right)$ and $\bar{M}$ is plotted. According to catastrophe theory, the stability changes at $d\left(1 / T_{H}\right) / d M=\infty[27-30]$ (see the Appendix). Since we cannot find the point $d\left(1 / T_{H}\right) / d M=\infty$ in the graph for various parameters, our solutions are stable in the catastrophic sense.

Finally, let us interpret a feature seen in Fig. 3. Seeing the relation between $\bar{M}$ and $\Psi_{H}$ for fixed $\Psi_{\infty}$ in Fig. 4, we find that $\Psi_{H}$ varies from a small value to 1 by reducing the mass. Thus, the separation between branes at the horizon is small for large mass; hence, the solution mimics the RN black hole. By reducing the mass, the separation goes to the infinity. Thus, $T_{H}$ eventually diverges like the GM-GHS solution as seen in Fig. 3.

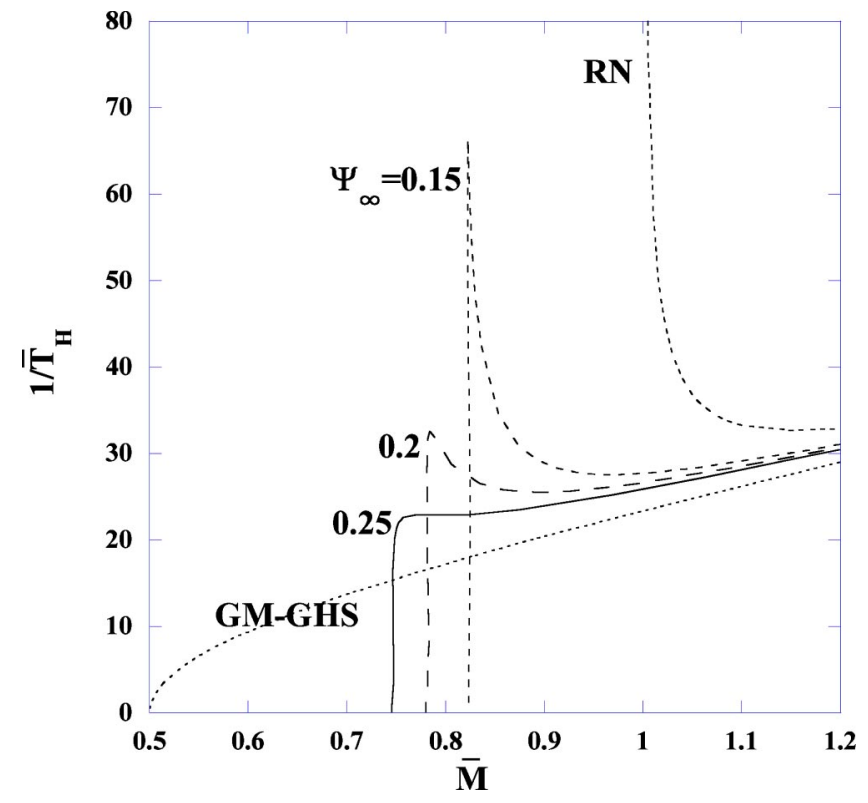

FIG. 3. $\bar{M}-1 / \bar{T}_{H}$ for $\bar{a}=\sqrt{3}$.

\section{VIEW FROM THE BULK}

Our interest here is the $y$ dependence of the horizon which had not been investigated so far in the RS1 model. The hologram (14) can be used to see the shape of the black strings. The differences from the previous papers [16-18] are that the matter is confined on the $\oplus$-brane and the branes are deformed by black strings. These differences will affect the shape of the horizon. Indeed, we will see that the black strings are nonuniform.

To see the nonuniformity of the horizon, let us investigate the change of the circumference radius along $y$ direction. The procedure to obtain a circumference radius of the horizon is summarized as follows: (i) Seek for the radius $r=r_{+}(y)$

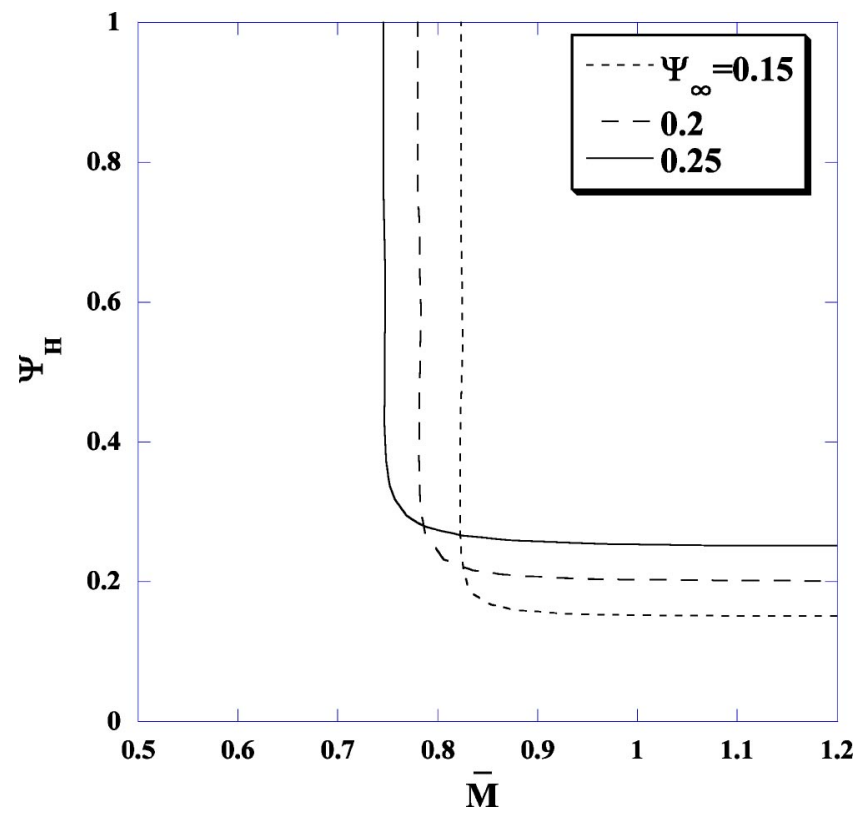

FIG. 4. $\bar{M}-\Psi_{H}$ for $\bar{a}=\sqrt{3}$. 


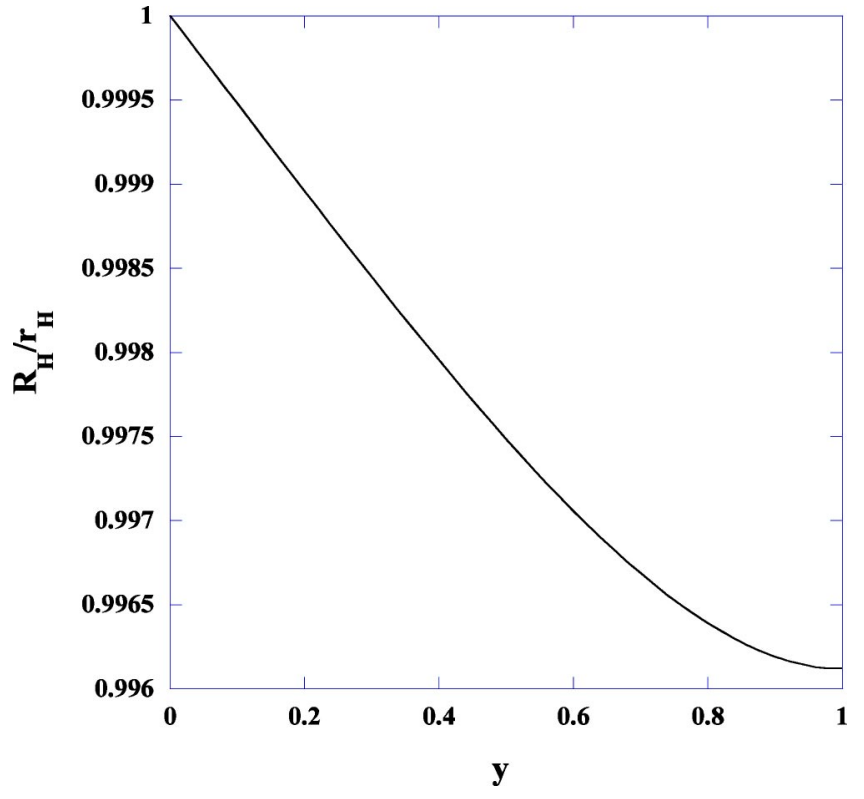

FIG. 5. Deformation of the horizon for $\Psi_{\infty}=0.25, \lambda_{H}=1.11$ $\times 10^{-2}, \bar{a}=\sqrt{3}$, and $\ell / r_{H}=0.5$.

which satisfies $h_{00}+g_{00}^{(1)}=0$ (or equivalently $h_{11}+g_{11}^{(1)}=\infty$ as we denote below). (ii) Evaluate the circumference radius in the Einstein frame as

$$
R_{H}:=\sqrt{r_{+}^{2}+\Psi\left(r_{+}\right) g_{22}^{(1)}\left(r_{+}\right)} .
$$

Note that we subtracted the effect of the AdS background in the above expression [see Eq. (14)].

First, let us find $r_{+}(y)$. Writing $g_{00}^{(1)}=: h_{00} f_{0}(r, y)$ and $g_{11}^{(1)}=: h_{11} f_{1}(r, y)$ and using Eq. (10), we can verify that $f_{0}$ $=f_{1}$ and they have finite values at $r=r_{H}$. Hence, the coordinate value of the horizon does not change $r_{+}(y)=r_{H}$ even at this order. Thus, we can evaluate the circumference radius (36) using the expression

$$
g_{22}^{(1)}\left(r_{H}\right)=-\frac{\ell^{2} w}{2}\left[R_{22}-\frac{1}{6} h_{22} R+(w+2) \chi_{22}\right] .
$$

Here, we introduced the variable $w:=1 / b^{2}-1$ which increases toward the $\ominus$-brane. Notice that $R_{22}$ and $R$ are those in the Jordan frame.

As an example, we show the ratio of the horizon, $R_{H} / r_{H}$, as a function of $y$ in Fig. 5. We find that the circumference radius of the horizon monotonically shrinks toward the $\ominus$-brane even if we subtract the background effect $b(y)$. Although we showed only one example, we can easily confirm that this is general by differentiating Eq. (37) with respect to $w$ since the fields satisfy the conditions

$$
T_{22}=\frac{\Psi Q_{m}^{2}}{2 r^{2}} e^{-2 a \phi}>0, T_{\mu}^{\mu}=0,
$$

at $r=r_{H}$, where $T_{22}$ and $T_{\mu}^{\mu}$ are those in the Jordan frame. In fact, by rewriting Eq. (37) as

$$
\begin{aligned}
K & :=-\frac{2}{\ell^{2}} g_{22}^{(1)}\left(r_{H}\right) \\
& =w\left[\frac{h_{22}}{3} R+\frac{\kappa^{2}}{\Psi} T_{22}\left\{1-(w+2) \frac{1-\Psi}{2}\right\}\right],
\end{aligned}
$$

we obtain

$$
\begin{aligned}
& \frac{d K}{d w}=\frac{h_{22}}{3} R+\frac{\kappa^{2}}{\Psi} T_{22}\{1-(w+1)(1-\Psi)\}, \\
& \frac{d^{2} K}{d w^{2}}=-\frac{\kappa^{2}}{\Psi}(1-\Psi) T_{22}<0 .
\end{aligned}
$$

From Eq. (41), we see that $d K / d w$ takes the minimum value at the $\ominus$-brane. On the $\ominus$-brane, we have

$$
\frac{d K}{d w}=\frac{1}{3} h_{22} R=-\frac{1}{3} h_{22} T_{\mu}^{\mu}=0 .
$$

Therefore, $d K / d w$ is always positive in the bulk. This means that $d g_{22}^{(1)} / d w<0$. As the horizon has nontrivial $y$ dependence, the black strings are nonuniform.

Numerically we found that the nonuniformity of the horizon becomes larger for solutions with smaller gravitational mass-i.e., smaller horizon radius.

Finally, we comment on the zeroth law of the nonuniform black string. Using the metric (18), the Hawking temperature $T_{H}$ is calculated as

$$
T_{H}=\frac{2 \pi-\kappa^{2} m_{H}^{\prime}}{8 \pi^{2} r_{H}} e^{-\delta_{H}} \sqrt{\frac{1+f_{0}\left(r_{H}, y\right)}{1+f_{1}\left(r_{H}, y\right)}} .
$$

Since $f_{0}=f_{1}$ at $r=r_{H}, T_{H}$ does not depend on $y$. Therefore, the zeroth law of black hole thermodynamics holds.

\section{CONCLUSION}

Nonuniform black strings in the two-brane system are investigated using the effective action approach. We considered the dilaton field coupled to the electromagnetic field on the $\oplus$-brane. It is shown that the radion acts as a nontrivial hair of the black strings. From the brane point of view, the black string appears as a deformed GM-GHS black hole which becomes a GM-GHS black hole in the single brane limit and reduces to a $\mathrm{RN}$ black hole in the close limit of two-branes. In view of catastrophe theory [27-30], our solutions are stable. From the bulk point of view, the black strings are proved to be nonuniform. Nevertheless, the zeroth law of black hole thermodynamics holds.

We established the picture shown in the upper right of Fig. 6. This picture shows that the event horizon shrinks toward the $\ominus$-brane (even if we subtract the effect of the AdS background) and the distance between branes decreases toward the asymptotically flat region. As the curvature on the brane increases, the nonuniformity of the horizon becomes larger. 
TABLE II. Catastrophic interpretation.

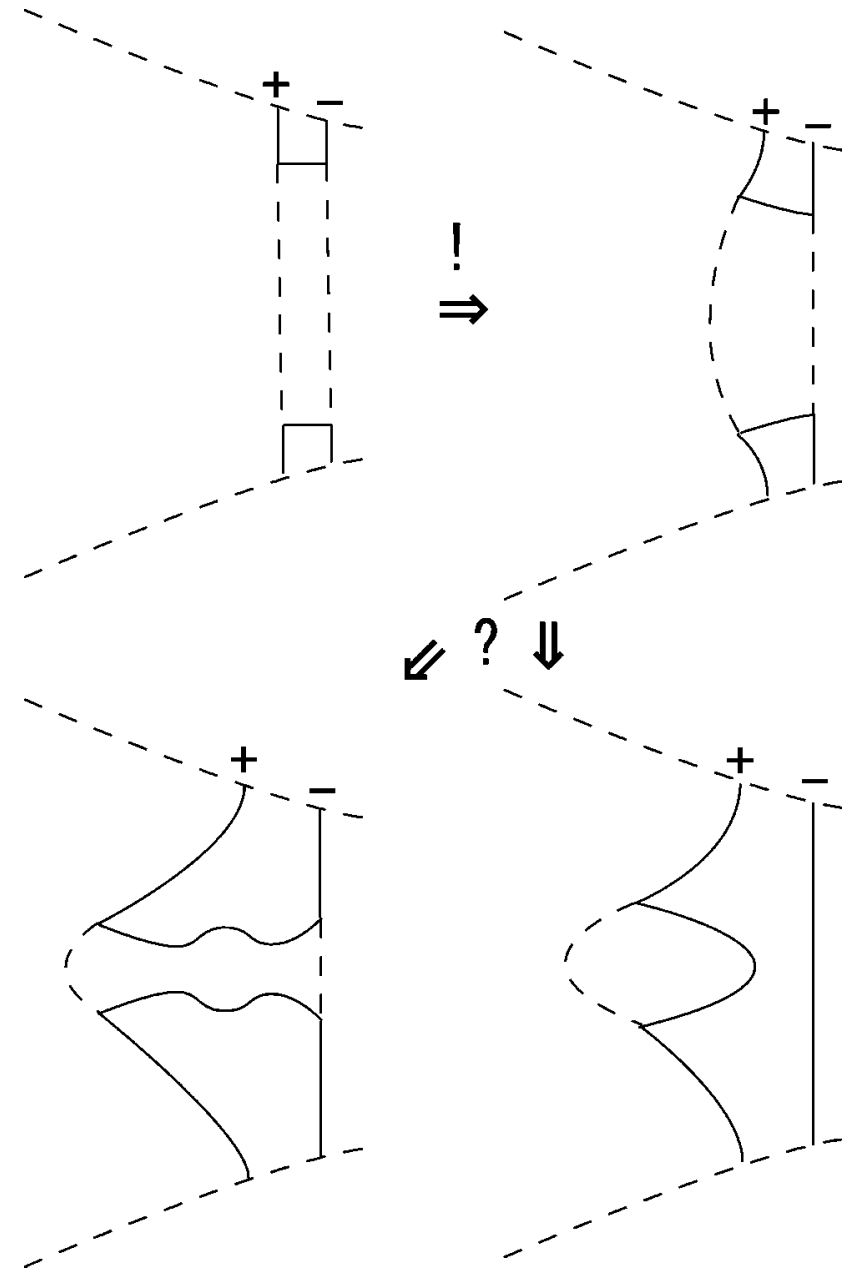

FIG. 6. Upper half is the image that we established in the text. Lower half shows two of the possibilities when the curvature on the brane becomes strong.

However, we cannot apply our present analysis if the distance between branes exceeds the horizon radius. This is because the Kaluza-Klein effect becomes significant. It is the point that Gregory-Laflamme instability commences. The transition to the localized black hole may occur as in the lower right of Fig. 6. The AdS/conformal field theory correspondence argument suggests classical evaporation of the resultant black hole [31]. Moreover, there is also a possibility that the shape of the horizon becomes complicated as in the lower left of Fig. 6. To get a hint, we need to proceed to the next order calculations corresponding to Kaluza-Klein corrections. We want to investigate it in the future.

\section{ACKNOWLEDGMENTS}

This work was supported in part by Grants-in-Aid for Scientific Research Fund of the Ministry of Education, Science and Culture of Japan No. 154568 (T.T.), No. 155476 (S.K.), and No. 14540258 (J.S.). This work was also supported in part by a Grant-in-Aid for the 21st Century COE Center for Diversity and Universality in Physics.

$$
\frac{\partial F(s, x)}{\partial x}=0 .
$$

Black hole mass $M$

Radion at asymptotic infinity $\Psi_{\infty}$ Magnetic charge $Q_{m}$

\begin{tabular}{lc} 
parameters & $\begin{array}{c}\text { Radion at asymptotic infinity } \Psi_{\infty} \\
\text { Magnetic charge } Q_{m}\end{array}$ \\
\hline Potential & Black hole entropy $A / 4$ \\
\hline \hline
\end{tabular}

\section{APPENDIX: CATASTROPHIC APPROACH}

We proposed the stability analysis of the black holes via catastrophe theory in previous papers $[28-30]$. We briefly summarize the discussion here. The potential function of the system is described by $F(s, x)$, where $s$ and $x$ are a control parameter and a state variable, respectively [32]. The control parameter means that the system is controlled by this parameter. The state variable is named for the reason that its value is determined after the state of the system is determined. Thom's theorem guarantees that if a system has control parameters $\leqslant 4$, the potential function can be made to coincide with one of the seven elementary catastrophe's potential functions by diffeomorphism.

The correspondence between catastrophic variables and black hole variables can be identified as in Table II. The equilibrium point of the system is written as

In the case of the black hole system, this corresponds to the static solution.

Let us find the catastrophic criterion of the stability of static solutions. We write $x$ which satisfies the condition (A1) as $x_{e q}(s)$. We also define $F_{e q}(s):=F\left(s, x_{e q}(s)\right)$. Then we obtain

$$
\frac{d^{2} F_{e q}(s)}{d s^{2}}=\left(\frac{\partial^{2} F}{\partial s^{2}}\right)_{e q}+\left(\frac{\partial^{2} F}{\partial x \partial s}\right)_{e q} \frac{d x_{e q}}{d s} .
$$

Because of the condition that $F_{e q}(s)$ be an equilibrium point, we also have

$$
0=\frac{d}{d s}\left(\frac{\partial F}{\partial x}\right)_{e q}=\left(\frac{\partial^{2} F}{\partial x \partial s}\right)_{e q}+\left(\frac{\partial^{2} F}{\partial x^{2}}\right)_{e q} \frac{d x_{e q}}{d s}
$$

If we eliminate $d x_{e q} / d s$ from Eq. (A2) using this equation, we obtain

$$
\frac{d^{2} F_{e q}(s)}{d s^{2}}=\left(\frac{\partial^{2} F}{\partial s^{2}}\right)_{e q}-\left(\frac{\partial^{2} F}{\partial x \partial s}\right)_{e q}^{2} /\left(\frac{\partial^{2} F}{\partial x^{2}}\right)_{e q}
$$

Since the point where stability changes corresponds to the inflection point of the potential function, we have $\left(\partial^{2} F / \partial x^{2}\right)_{e q}=0$. Then, unless 


$$
\left(\frac{\partial^{2} F}{\partial x \partial s}\right)_{e q}=0,
$$

we have

$$
\frac{d^{2} F_{e q}(s)}{d s^{2}}=\infty .
$$

We apply the formula (A4) to black holes. If we fix $\Psi_{\infty}$ and $Q_{m}, d F_{e q} / d s$ corresponds to $d(A / 4) / d M$ and equals $1 / T_{H}$ from the first law of black hole thermodynamics. Because of Eq. (A6), stability changes at

$$
\frac{d\left(1 / T_{H}\right)}{d M}=\infty .
$$

Therefore, what we should do is to examine if the point where Eq. (A7) holds exists or not for various $\Psi_{\infty}$ and $Q_{m}$. We have already known that the GM-GHS solutions are stable at least. Then, if no such a point is found, the solutions are stable in the catastrophic sense.

There may be a possibility that Eq. (A5) holds. In such a case, the argument based on catastrophe theory fails. However, the argument of the inner horizon supports the belief that it is not the case.
[1] P. Horava and E. Witten, Nucl. Phys. B460, 506 (1996); B475, 94 (1996).

[2] L. Randall and R. Sundrum, Phys. Rev. Lett. 83, 4690 (1999); 83, 3370 (1999).

[3] K. Koyama and J. Soda, Phys. Lett. B 483, 432 (2000); Phys. Rev. D 62, 123502 (2000); 65, 023514 (2002); N. Kaloper, ibid. 60, 123506 (1999); H.B. Kim and H.D. Kim, ibid. 61, 064003 (2000); G.R. Maartens, D. Wands, B.A. Bassett, and I. Heard, ibid. 62, 041301(R) (2000); H. Kodama, A. Ishibashi, and O. Seto, ibid. 62, 064022 (2000); C. van de Bruck, M. Dorca, R.H. Brandenberger, and A. Lukas, ibid. 62, 123515 (2000); D. Langlois, R. Maartens, M. Sasaki, and D. Wands, ibid. 63, 084009 (2001); H.A. Chamblin and H.S. Reall, Nucl. Phys. B562, 133 (1999); D. Langlois, Phys. Rev. Lett. 86, 2212 (2001).

[4] A. Chamblin, S.W. Hawking, and H.S. Reall, Phys. Rev. D 61, 065007 (2000).

[5] A. Chamblin, H.S. Reall, H. Shinkai, and T. Shiromizu, Phys. Rev. D 63, 064015 (2001).

[6] T. Shiromizu and M. Shibata, Phys. Rev. D 62, 127502 (2000).

[7] R. Emparan, G.T. Horowitz, and R.C. Myers, J. High Energy Phys. 01, 007 (2000); 01, 021 (2000).

[8] H. Kudoh, T. Tanaka, and T. Nakamura, Phys. Rev. D 68, 024035 (2003); H. Kudoh, hep-th/0306067.

[9] P. Kanti and K. Tamvakis, Phys. Rev. D 65, 084010 (2002); P. Kanti, I. Olasagasti, and K. Tamvakis, ibid. 68, 124001 (2003).

[10] N. Dadhich, R. Maartens, P. Papadopoulos, and V. Rezania, Phys. Lett. B 487, 1 (2000); M. Bruni, C. Germani, and R. Maartens, Phys. Rev. Lett. 87, 231302 (2001); R. Casadio and L. Mazzacurati, Mod. Phys. Lett. A 18, 651 (2003); R. Casadio, A. Fabbri, and L. Mazzacurati, Phys. Rev. D 65, 084040 (2002); K.A. Bronnikov, V.N. Melnikov, and H. Dehnen, ibid. 68, 024025 (2003).

[11] S. Kanno and J. Soda, Phys. Rev. D 66, 083506 (2002); 66, 043526 (2002); Astrophys. Space Sci. 283, 481 (2003); J. Soda and S. Kanno, ibid. 283, 639 (2003); S. Kanno and J. Soda, hep-th/0303203.

[12] S. Kanno, M. Sasaki, and J. Soda, Prog. Theor. Phys. 109, 357 (2003).

[13] G.W. Gibbons and K. Maeda, Nucl. Phys. B298, 741 (1988); D. Garfinkle, G.T. Horowitz, and A. Strominger, Phys. Rev. D 43, 3140 (1991).

[14] C.F.E. Holzhey and F. Wilczek, Nucl. Phys. B380, 447 (1992).
[15] R. Gregory and R. Laflamme, Phys. Rev. Lett. 70, 2837 (1993).

[16] G.T. Horowitz and K. Maeda, Phys. Rev. Lett. 87, 131301 (2001).

[17] T. Wiseman, Class. Quantum Grav. 20, 1137 (2003); 20, 1177 (2003); B. Kol and T. Wiseman, ibid. 20, 3493 (2003).

[18] M. Choptuik et al., Phys. Rev. D 68, 044001 (2003).

[19] T. Wiseman, Class. Quantum Grav. 19, 3083 (2002); T. Chiba, Phys. Rev. D 62, 021502(R) (2000); P. Brax, C. van de Bruck, A.C. Davis, and C.S. Rhodes, ibid. 67, 023512 (2003); T. Shiromizu and K. Koyama, ibid. 67, 084022 (2003).

[20] K. Tomita, Prog. Theor. Phys. 54, 730 (1975); J. Soda, H. Ishihara, and O. Iguchi, ibid. 94, 781 (1995); G.L. Comer, N. Deruelle, D. Langlois, and J. Parry, Phys. Rev. D 49, 2759 (1994).

[21] T. Shiromizu, K. Maeda, and M. Sasaki, Phys. Rev. D 62, 024012 (2000).

[22] G. Gibbons, R. Kallosh, and B. Kol, Phys. Rev. Lett. 77, 4992 (1996).

[23] R. M. Wald, General Relativity (The University of Chicago Press, Chicago, 1984).

[24] T. Okai, Class. Quantum Grav. 11, 409 (1994).

[25] The value $\bar{a}=\sqrt{3}$ is favored in Kaluza-Klein theory. However, our explanation does not depend on the dilaton coupling qualitatively.

[26] J.D. Bekenstein, Phys. Rev. D 5, 1239 (1972); S.W. Hawking, Commun. Math. Phys. 25, 167 (1972).

[27] O. Kaburaki, I. Okamoto, and J. Katz, Phys. Rev. D 47, 2234 (1993); J. Katz, I. Okamoto, and O. Kaburaki, Class. Quantum Grav. 10, 1323 (1993).

[28] K. Maeda, T. Tachizawa, T. Torii, and T. Maki, Phys. Rev. Lett. 72, 450 (1994); T. Torii, K. Maeda, and T. Tachizawa, Phys. Rev. D 51, 1510 (1995); T. Tachizawa, K. Maeda, and T. Torii, ibid. 51, 4054 (1995).

[29] T. Tamaki, K. Maeda, and T. Torii, Phys. Rev. D 57, 4870 (1998); 60, 104049 (1999).

[30] T. Tamaki, T. Torii, and K. Maeda, Phys. Rev. D 68, 024028 (2003).

[31] T. Tanaka, Prog. Theor. Phys. Suppl. 148, 307 (2002); R. Emparan, A. Fabbri, and N. Kaloper, J. High Energy Phys. 08, 043 (2002).

[32] T. Poston and I. Stewart, Catastrophe Theory and Its Applications (Pitmann, London, 1978); R. Thom, Structure Stability and Morphogenesis (Benjamin, New York, 1975). 